\title{
Connectivity in one-dimensional ad hoc networks: A queueing theoretical approach
}

\author{
Daniele Miorandi · Eitan Altman
}

Published online: 8 May 2006

(C) Springer Science + Business Media, LLC 2006

\begin{abstract}
In this paper we analyze connectivity issues in one-dimensional ad hoc networks. Starting with a deterministic channel model, we show how an equivalent $G I|D| \infty$ queueing model may be used to address network connectivity. In this way, we obtain exact results for the coverage probability, the node isolation probability and the connectivity distance for various node placement statistics. We then show how a $G I|G| \infty$ model may be used to study broadcast percolation problems in ad hoc networks with general node placement in the presence of fading channels. In particular, we obtain explicit results for the case of nodes distributed according to a Poisson distribution operating in a fading/ shadowing environment. In the latter case, heavy traffic theorems are applied to derive the critical transmission power for connectivity and broadcast percolation distance in highly dense networks. The impact of signal processing schemes able to exploit the diversity provided by smallscale fading by means of multiple antennas is considered. The analysis is then extended to the case of unreliable ad hoc networks, with an in-depth discussion of asymptotic results.
\end{abstract}

This work was partially supported by the EURO NGI Network of Excellence. The work of D. Miorandi was partially supported by Fond. A. Gini. This work has been done while D. Miorandi, at that time with University of Padova (Italy), was visiting the MAESTRO project at INRIA Sophia Antipolis.

D. Miorandi $(\square)$

CREATE-NET, v. Solteri 38, 38100, Trento, Italy

e-mail: daniele.miorandi@create-net.it

E. Altman

INRIA, 2004 Route des Lucioles, 06902, Sophia Antipolis, France

e-mail: eitan.altman@sophia.inria.fr
Keywords Ad hoc networks - Connectivity . $G I|G| \infty$. Broadcast percolation · Fading channels · Diversity techniques

\section{Introduction}

The growing interest in the field of self-organizing wireless networks, often referred to as ad hoc, has led to a considerable amount of literature dealing with the characterization of the limiting performance of such networks, in terms of both connectivity [1-4] and capacity [5-10], two intimately related issues [11]. In this paper, we focus on one-dimensional ad hoc networks, in which nodes are randomly deployed over an infinite line, and present novel results on connectivity and coverage. These results are obtained by means of an equivalent infinite-server queueing model. One-dimensional networks are interesting since, on the one hand, they represent a meaningful model for some applications (e.g., car networks), while, on the other one, their analysis may provide insight into more complex two-dimensional networks. Further, the results we get in the one-dimensional case may be used to obtain bounds on the connectivity of networks in higher dimensions, although the resulting bounds are known not to be tight $[2,12]$.

In the literature, results for the connectivity of onedimensional networks are presented in $[2,3,12,13]$. In all these papers, the nodes are assumed to be distributed according to either a uniform or a Poisson distribution on an infinite line. In this paper, we remove this assumption, and obtain results for the case of general inter-node distance. Further, in all the aforementioned papers the authors consider a (simplistic) deterministic model for radio propagation. In this work, we show how we can enhance the model, in order to account 
for the presence of shadowing and/or fading phenomena on the network connectivity. Note that, in a two-dimensional framework, some results on the impact of channel randomness are known [14-16]. On the other hand, to the best of authors knowledge, no in-depth analysis of connectivity with node placement distributions other than uniform or Poisson has been presented so far. Further, note that, according to the results in $[2,17]$, we expect our analysis to hold with a good degree of approximation also for the case of mobile ad hoc networks in the presence of a random waypoint mobility model.

The relationship between coverage problems and infiniteserver queues is an old and well-established one [18]. In the paper, we show how this link can be usefully exploited and, in particular, how, using some results on the busy period distribution of $G I|G| \infty$ queues, extensions to previously published results on the connectivity properties of ad hoc networks may be found.

In the presence of a deterministic model for radio propagation, we obtain closed-form results for the connectivity distance in networks with general node placement statistics, and analyze the performance obtained with different distributions of the inter-node distance. We also provide an analytical characterization of other performance metrics, such as the coverage probability and the node isolation probability. A comparison between various node placement statistics is carried out, and some remarks on the limiting behavior in very dense networks are presented. These results are obtained using results from the theory of $G I|D| \infty$ queues.

By using a more general $G I|G| \infty$ framework, we can easily account for various channel impairments (e.g., shadowing, fading). When we account for random channels, the notion of connectivity fades, due to the different propagation conditions that may be encountered in the forward and backward directions. In this case, we focus on the problem of broadcast percolation, where propagation of one message in the forward direction is studied. In this case, closed-form results may be obtained for the case of nodes distributed accorded to a Poisson point process. We focus on this distribution in order to keep the tractation simple and to gain insight into the impact of channel randomness on the connectivity properties of the resulting network. The effect of lognormal shadowing and Rayleigh fading is analyzed and discussed.

Heavy traffic limits are used to characterize the asymptotic behavior in very dense networks; results for the critical transmission power for both connectivity and broadcast percolation are provided. In particular, the critical transmission range in the $M|D| \infty$ model turns out to be the same found with a different approach in [1].

The analysis is also extended to the case of multiple antennas, where the diversity provided by the radio channel is exploited to enhance the system performance. The performance of two classical signal processing techniques, best path selection and maximal ratio combining, is analyzed in the presence of Rayleigh fading. It is shown that, even with two antennas, the use of such algorithms leads to a performance improvement with respect to a deterministic channel model, thus showing how the diversity provided by fading may be exploited to improve network connectivity. Finally, we study the impact of node failures on connectivity performance. A general discussion of how to incorporate this issue into the general framework is provided, and an in-depth asymptotic analysis is presented. In particular, for the $M|D| \infty$ case, results equivalent to those presented in [19] for grid networks are found.

The paper is organized as follows: Section 2 presents connectivity results for a deterministic channel model. In Section 3 the broadcast percolation problem in the presence of channel randomness is addressed. Section 4 presents results on network connectivity in the presence of unreliable devices. Section 5 concludes the paper with a brief summary of the results and some open issues.

\section{A $G I|D| \infty$ model for connectivity in one-dimensional ad hoc networks}

Let us consider a one-dimensional network, where nodes are randomly placed along a semi-infinite line. Let us denote by $X_{n}$ the position of the $n$-th node, with $X_{0}=0$. Further, we denote by $Y_{n}=X_{n+1}-X_{n}$ the distance between two successive nodes. We assume that $\left\{Y_{n}\right\}_{n \in \mathbb{N}}$ is a sequence of independent identically distributed (iid) random variables, so that, in the following, we drop the index $n$. We start by considering the simplest model for signal propagation, where only path loss is present, so that power decays with distance as $d^{-\alpha}$, where $\alpha \geq 2$. Further, we assume that "good long" codes are used, so that the probability of a successful packet reception, as a function of the signal-to-noise ratio (SNR), approaches a step function, whose threshold is denoted by $\Psi$. Further, we assume that an additive Gaussian noise of power $P_{\text {noise }}$ is present at the receiver. Denoting by $P_{t x}$ the transmission power, we find that two nodes may communicate with each other if their distance is less than or equal to the communication range $R=\left(\frac{P_{t x}}{\Psi P_{\text {noise }}}\right)^{\frac{1}{\alpha}}$. In such a situation it is a well known result that, for any finite $R$, the resulting network will be disconnected P-almost surely [3]. Furthermore, the network will be $\mathrm{P}$-almost surely divided into an infinite number of finite clusters, which will be referred to in the following as "spatial clusters". In order to characterize the cluster statistics, let us consider an equivalent $G I|D| \infty$ model, with inter-arrival times distributed as $Y$ and deterministic service time $R$, as depicted in Fig. 1. Then, a spatial cluster in the ad hoc network corresponds to a busy period in the queueing 


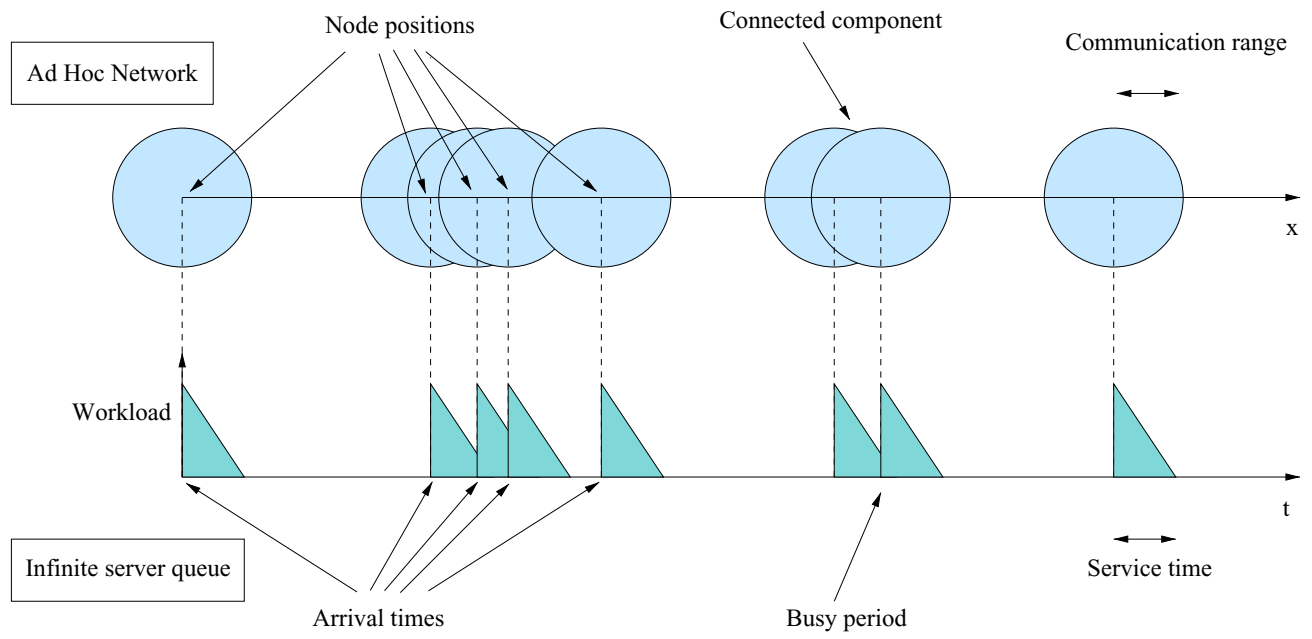

Fig. 1 Ad hoc networks with deterministic transmission range and equivalent $G I|D| \infty$ queueing model

model. Accordingly, the length of the connected component corresponds to the busy period duration, and the number of nodes in a cluster corresponds to the number of customers served during a busy period. In the sequel of the paper, we will use the ad hoc network terminology.

\subsection{Cluster size and connectivity distance}

Let $F_{Y}(a)$ and $f_{Y}(a)$ be the cumulative distribution function (cdf) and the probability density function (pdf) of the internode distance $Y$, respectively. Further, let $\lambda$ be the average node density, $\lambda=\frac{1}{E[Y]}$. We denote by $B_{n}$ and $N_{n}$ the duration of the $n$-th connected component and the number of devices therein; both are iid.

We define as connectivity distance the size of the first connected component. Since $X_{0}=0$, this corresponds to the distance within which devices are able to communicate with the node located at the origin.

The Laplace-Stieltjes transform (LST) of the connectivity distance is given by [20]:

$\mathcal{B}(s)=\frac{e^{-s R}\left(1-F_{Y}(R)\right)}{1-\int_{0}^{R} e^{-s t} f_{Y}(t) \mathrm{d} t}$.

While in general this expression may not be inverted directly, and one has to resort to numerical methods [21], the moments of any order may easily be computed by differentiation. For example, the average connectivity distance is given by:

$E[B]=-\left.\frac{\partial \mathcal{B}(s)}{\partial s}\right|_{s=0}=R+\frac{\int_{0}^{R} t f_{Y}(t) \mathrm{d} t}{1-F_{Y}(R)}$

Accordingly, the probability generating function (pgf) of the number $N$ of devices forming a spatial cluster is given by [20]:

$\mathcal{N}(z)=z \frac{1-F_{Y}(R)}{1-z F_{Y}(R)}$.

Inverting, we find the probability mass function (pmf) of the random variable $N$, which is geometrically distributed:

$$
P[N=k]=\left(1-F_{Y}(R)\right) \cdot F_{Y}(R)^{(k-1)}, \quad k=1,2, \ldots
$$

The average number of connected devices is given by:

$E[N]=\frac{1}{1-F_{Y}(R)}$.

The probability that at least $k$ devices are connected, may be obtained as:

$p_{R}(k)=P[N \geq k]=\sum_{t=k}^{\infty} P[N=t]=F_{Y}(R)^{(k-1)}$.

Some results, for the case of exponential distribution, are reported in Fig. 2, where the probability that the $k$-th node is connected to the first one is depicted for $k$ ranging from 10 to 100 as a function of the average number of nodes in range, $\lambda R$.

The problem of connectivity in finite ad hoc networks was treated for the first time in [12], where the authors focus on a network with $n$ nodes placed according to a Poisson process. We work in a slightly different framework, considering the probability of being able to connect to a device located at distance $d$ from the origin. This may be obtained considering the distribution of the connectivity distance:

$p_{C}(d)=P[B>d]=1-F_{B}(d)$

In general, to obtain the complementary cumulative distribution function (ccdf) of the connectivity distance, one 


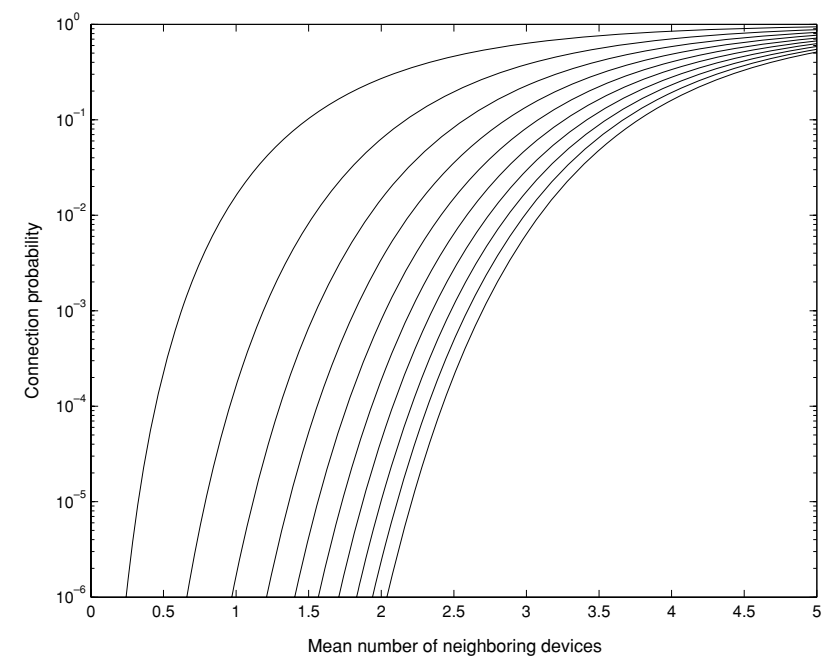

Fig. $2 k$-th node connection probability $(k=10,20, \ldots, 100$ from the left to the right) vs. $\lambda R$, Poisson distribution of nodes

should perform the inversion of the pdf LST given in (1) or, directly, of the ccdf $\operatorname{LST} \hat{\mathcal{B}}(s)=\frac{1-\mathcal{B}(s)}{s}$. One case of practical interest, where direct inversion of the LST is possible, is that of nodes distributed according to a Poisson distribution, with average density $\lambda$. In this case the pdf LST is given by:

$\mathcal{B}(s)=\frac{(s+\lambda) e^{-(s+\lambda) R}}{s+\lambda e^{-(s+\lambda) R}}$,

whose inversion leads to [22,23]:

$$
\begin{gathered}
f_{B}(t)=e^{-\lambda R} \delta(t-R)+1(t-R) \cdot \lambda e^{-\lambda R} \sum_{k=0}^{\left\lfloor\frac{t}{b}\right\rfloor-1}\left(-\lambda e^{-\lambda R}\right)^{k} \\
\times \frac{[t-R(k+1)]^{k}-e^{-\lambda R} \cdot \max \left\{0,[t-R(k+2)]^{k}\right\}}{k !},
\end{gathered}
$$

where $\delta(\cdot)$ is the Dirac delta function and $1(\cdot)$ represents the unit step function. Then, evaluation of $p_{C}(d)$ may be performed integrating (8), leading to the formula obtained in [3] solving a differential equation with appropriate boundary conditions.

In Fig. 3 we plotted (on a logarithmic scale) the probability that a node placed at a distance $d$ from the origin is connected for various values of the node density $\lambda$, with $R=1 \mathrm{~m}$. Note that, for the values of $\lambda$ considered, a small variation of the node density leads to a great variation in the probability that a node at distance $d$ is connected. In order to get more insight into such phenomena, we showed in Fig. 4 the probability of being connected at distances $d=10,50,100 \mathrm{~m}$ vs. the node density $\lambda$ for the case $R=1$. For the parameters considered, which define a medium density network, the curves show a gentle behavior, suggesting the absence of criticality phenomena. In Fig. 5 we plotted the probability of connection

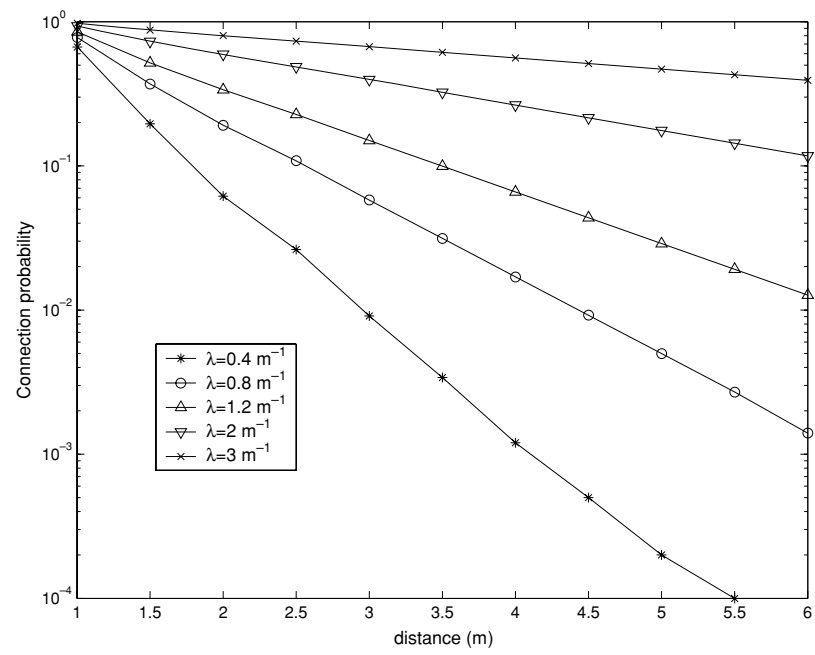

Fig. 3 Probability of connection for a node placed at $d$ for different values of $\lambda, R=1 \mathrm{~m}$, Poisson distribution of nodes

at a distance $d=1 \mathrm{~m}$ vs. the communication range $R$ for various values of $\lambda$. From the curves it is apparent that a phase transition, i.e. an abrupt change from a very low connection probability to a very high value of such parameter, takes place for the case of very large node density. Such critical connectivity phenomena $[1,12,13,24]$ do not arise in the presence of a low-to-medium value of the node density $\lambda$.

\subsection{Coverage and isolation probability}

In sensor networks, an important performance metric is the coverage probability, which reflects the probability that a point of the network is "covered" by at least one sensor. Let us assume that sensors have a range of $\frac{R}{2}$ and denote by $p_{c o v}$ the probability of the network being covered. Since the arrival process is stationary, a translation of $\frac{R}{2}$ does not

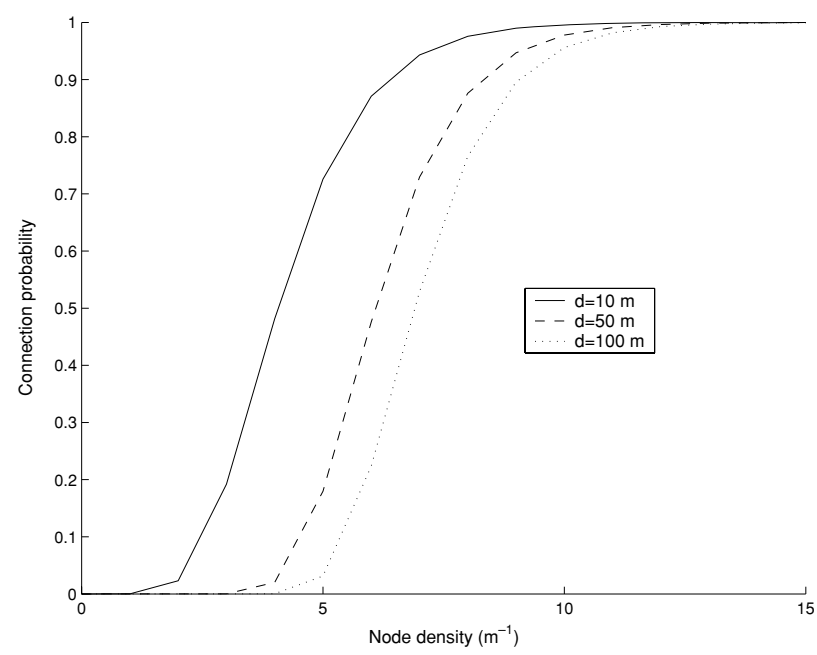

Fig. 4 Probability of connection at $d$ vs. $\lambda, R=1 \mathrm{~m}$, Poisson distribution of nodes 


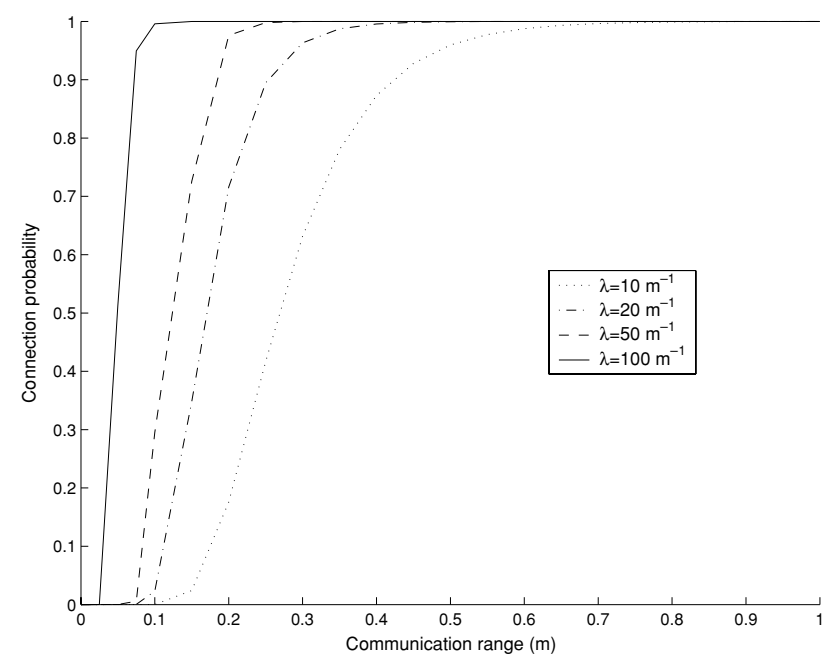

Fig. 5 Probability of connection at $d=1 \mathrm{~m}$ vs. $R$ for various node densities, Poisson distribution of nodes

change $p_{\text {cov }}$; then we may apply our $G I|D| \infty$ model with service time equal to $R$. In the evolution of the system, it is easy to recognize the presence of renewal points, which correspond to the beginning of a busy period. Since the system is stationary ergodic, the probability that a point is covered corresponds to the fraction of the busy cycle, whose length is denoted by $C$, during which the system is busy. Hence, $p_{c o v}=\frac{E[B]}{E[C]}$. Then, applying results from [20], we get:

$p_{c o v}=\frac{E[B]}{E[C]}=\lambda R-\lambda \int_{0}^{R}(R-a) f_{Y}(a) \mathrm{d} a$.

Another parameter of interest is the probability $p_{I}$ that a given device is isolated:

$p_{I}=P[N=1]=1-F_{Y}(R)$.

Comparing (10) with (5), we note that $p_{I}=\frac{1}{E[N]}$. Again, from (6) and (10), it is apparent that we may write $p_{R}(k)=$ $\left(1-p_{I}\right)^{(k-1)}$.

It is worth remarking that there is an interesting relationship linking $p_{I}$ and $p_{c o v}$. Indeed, the node isolation probability may be thought as the complement of the probability of the event $A=\{$ being covered $\}$ restricted to the point process $\left\{X_{n}\right\}$. In this view, it is clear that we can think of $p_{I}$ as the Palm probability [25] of the complement of $A$ associated to the point process $\left\{X_{n}\right\}$. This relationship does not depend on the communication range statistics and holds for a general $G|G| \infty$ model. Thus, we can link the two quantities through the following Palm inversion formula [25]:

$p_{\text {cov }}=P[A]=\lambda \int_{0}^{+\infty} \mathrm{d} t P^{0}\left[Y_{1}>t, \theta_{t} \in A\right]$, where $P^{0}$ denotes the Palm probability associated to $\left\{X_{n}\right\}$.

Specifying (11) to the $G I|D| \infty$ case and denoting by $\chi\{\cdot\}$ the indicator function of a given event, we get:

$$
\begin{aligned}
p_{c o v} & =\lambda \int_{0}^{+\infty} \mathrm{d} t P[Y>t] \chi\{t \leq R\}=\lambda \int_{0}^{R} \mathrm{~d} t\left[1-F_{Y}(t)\right] \\
& =\lambda R-\int_{0}^{R} \mathrm{~d} t \int_{0}^{t} \mathrm{~d} a f_{Y}(a)=\lambda R-\int_{0}^{R} \mathrm{~d} a \int_{a}^{R} \mathrm{~d} t f_{Y}(a) \\
& =\lambda R-\int_{0}^{R} \mathrm{~d} a(R-a) f_{Y}(a),
\end{aligned}
$$

thus retrieving (9). Note that in the derivation above we exploited the fact that, in a renewal process, the distribution of the sequence $\left\{X_{n}\right\}$ is the same under $P$ and $P^{0}$ [25].

Further, by means of Slivnyak's theorem [25], we can state that, if $\left\{X_{n}\right\}$ forms a Poisson point process, then $p_{\text {cov }}=$ $1-p_{I}$, regardless of the communication range statistics.

The probability $p_{I}$ may be taken as a meaningful metric for comparing node placement distributions. As an example, in Fig. 6 we plotted $p_{I}$ vs. the mean distance between adjacent nodes for four different node placement statistics for $R=1 \mathrm{~m}$. We compared the following distributions for the distance between neighboring nodes: uniform, exponential, normal (with the natural scaling $\sigma^{2}=\lambda^{2}$ ) and Pareto (with a shape factor $\beta=1.5$ ). For very low node density, less than $0.5 \mathrm{~m}^{-1}$, the four curves are close together; in any case such a case is of low interest for application purposes, since the nodes are isolated with high probability. In the presence of a medium node density, corresponding to $\lambda$ ranging from 0.5 to 2.5 , we note the very good performance attained by the Pareto distribution, which overcomes the exponential one. A sharp transition is presented by the uniform distribution, which annihilates the node isolation probability for $\lambda>2 \mathrm{~m}^{-1}$. The

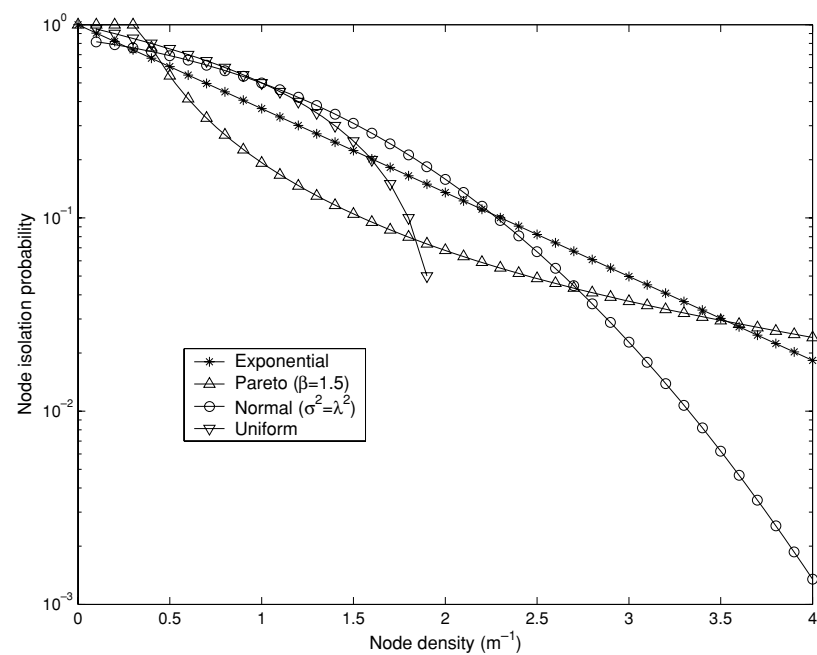

Fig. 6 Node isolation probability vs. average node density, $R=1 \mathrm{~m}$ for various node placement statistics 
normal distribution (which corresponds to the case of nodes placed one after the other, the distance being deterministic in principle but affected by a Gaussian placement error), on the other hand, behaves poorly in the medium density regime, while showing very good performance in a very dense network. In order to better understand the performance in very dense networks, let us consider the following heuristic. In a very dense network, i.e. when $\lambda R \gg 1$, the probability that a node is isolated is vanishing. From (10), this corresponds to a situation where the cdf of the random variable $Y$ is close to 1 . In some sense, we are actually looking at the tail of the distribution of the inter-node distance. Then, the heavier the tail of such distribution, the worse the performance in the presence of a very large node density.

In some envisioned applications (e.g. mobile military networks or highway networks), ad hoc networks are expected to be constituted by a massively large number of devices, so that the study of their limiting behavior in presence of very high node density is of interest. Scaling the mean distance between nearby nodes as $\frac{1}{\lambda}$ and taking $\lambda \rightarrow+\infty$, we are interested in finding a scaling of $R(\lambda)$ which ensures that the node isolation probability decays sufficiently fast (in our case, as $\frac{1}{\lambda}$ ). This enables us to compare different node distribution statistics in very dense networks.

For the exponential distribution, we have $p_{I}=e^{-\lambda R(\lambda)}$, so that:

$R(\lambda)^{\exp }=\frac{\ln \lambda}{\lambda}$.

For the Pareto distribution, with shape parameter $\beta$, the node isolation probability is given by $p_{I}=\left(\frac{\beta-1}{\beta \lambda R(\lambda)}\right)^{\beta}$. Thus,

$R(\lambda)^{P a r}=\frac{\lambda^{\frac{1}{\beta}}}{\lambda}$.

For the Gaussian distribution, we have, for the natural scaling of the variance $\sigma^{2}=\sigma_{0}^{2} \lambda^{-2}$, that the node isolation probability is given by $p_{I}=Q\left(\frac{\lambda R(\lambda)-1}{\sigma_{0}}\right)$. Since $Q(z) \sim e^{-\frac{z^{2}}{2}}$ for $z \rightarrow+\infty$, we have:

$R(\lambda)^{\text {norm }}=\frac{\sqrt{\ln \lambda}}{\lambda}$

For the uniform distribution, $p_{I}=\frac{2-\lambda R(\lambda)}{2}$, and thus:

$R(\lambda)^{\text {unif }}=\frac{2(\lambda-1)}{\lambda^{2}} \sim \frac{1}{\lambda}$.

As it might be expected, the distribution with no tail (uniform) outperforms all the others, whose performance confirms the heuristics discussed above on the impact of the tail of the distribution on the connectivity performance.

\subsection{Heavy traffic analysis for dense networks}

Let us consider the limiting behavior of an $M|D| \infty$ network under heavy traffic; this corresponds to a network with a very high density of nodes. For such a system, the busy period, appropriately scaled, converges to an exponential distribution [23]:

Theorem 1. As $\lambda \rightarrow+\infty$,

$F_{B}(a E[B]) \rightarrow 1-e^{-a}, a>0$.

Since the average busy period length is given by $E[B]=$ $\frac{e^{\lambda R}-1}{\lambda}$, we have that a node at distance $d$ is connected with probability

$p_{C}(d)=P[B>d]=e^{-\frac{d}{E[B]}}=e^{-\frac{d \lambda}{e^{\lambda R}-1}}$.

Thus, we may focus on the behavior of the function $f(\lambda)=\frac{d \lambda}{e^{\lambda}-1}$. If $f(\lambda) \rightarrow 0$, then the network is asymptotically connected ${ }^{1}$ at any finite distance $d$. Now, let us assume that the transmission range scales with $\lambda$ as $[1,13]$ :

$R(\lambda)=\frac{\ln \lambda+c(\lambda)}{\lambda}$.

Then, $f(\lambda)=\frac{0 d \lambda}{e^{(\lambda) \lambda} \lambda-1}$. Taking the limit for $\lambda \rightarrow+\infty$, we get:

$\lim _{\lambda \rightarrow+\infty} f(\lambda)=\frac{d}{e^{c}}$,

where $c=\lim _{\lambda \rightarrow+\infty} c(\lambda)$. Thus, for any given $d$, a necessary and sufficient condition for asymptotic connectivity is that $c=+\infty$. Hence, we get an analogous of the result of Gupta and Kumar [1]:

Theorem 2. Given a one-dimensional ad hoc network, with nodes placed according to a Poisson point process of intensity $\lambda$ and having a deterministic transmission range $R(\lambda)=\frac{\ln \lambda+c(\lambda)}{\lambda}$, asymptotic connectivity at any finite distance $d$ is ensured as $\lambda \rightarrow+\infty$ iff $\lim _{\lambda \rightarrow+\infty} c(\lambda)=+\infty$.

It is worth remarking that the condition for asymptotic connectivity does not depend on the distance $d$, if the latter is finite. This may be understood by the fact that asymptotic connectivity, in a one-dimensional setting, corresponds to the arising of an infinite component.

Similarly, we may get conditions on the communication range in order to ensure that the node isolation probability

\footnotetext{
${ }^{1}$ A network is said to be asymptotically connected if the connection probability vanishes as the node density $\lambda$ tends to infinity.
} 
goes to zero. As it may be easily expected, it turns out that the conditions are different, the one for connectivity being stronger, which shows that the widespread use of a sort of equivalence in the asymptote for the two concepts may lead to erroneous results.

Theorem 3. Given a one-dimensional ad hoc network, with nodes placed according to a Poisson point process of intensity $\lambda$ and having a deterministic transmission range $R(\lambda)=\frac{\ln \lambda+c(\lambda)}{\lambda}$, the absence of isolated nodes is asymptotically ensured as $\lambda \rightarrow+\infty \operatorname{iff}^{2} e^{-c(\lambda)}=o(\lambda)$.

Proof: The probability of a node being isolated is given by $p_{I}=e^{-\lambda R(\lambda)}$. Taking the limit, we get:

$\lim _{\lambda \rightarrow+\infty} p_{I}=\lim _{\lambda \rightarrow+\infty} \frac{e^{-c(\lambda)}}{\lambda}$.

Trivially, we may conclude that the absence of isolated nodes is asymptotically ensured for any finite $c=$ $\lim _{\lambda \rightarrow+\infty} c(\lambda)$. Note that the asymptote of $p_{I}$ can be readily computed for any distribution. Even if the disappearance of isolated nodes is only a loose necessary condition for network connectivity, a tighter condition can be found for the exponential case.

Proposition 1. Given that nodes are distributed according to a Poisson point process, a necessary and sufficient condition for asymptotic connectivity at any finite distance $d$ is that $\lim _{\lambda \rightarrow+\infty} \lambda p_{I}=0$.

Proof: It suffices to substitute the expression for the exponential distribution and compare with Theorem 2.

It is worth remarking that, while all the above results have been given in terms of "critical transmission range", they can be easily restated in terms of "critical transmission power". Indeed, the scaling for $P_{t x}$ is of the form $P_{t x}(\lambda)=\Psi P_{\text {noise }}\left(\frac{\ln \lambda+c(\lambda)}{\lambda}\right)^{\alpha}$. We will see in the following that the same scaling law holds in the case of shadowing/ fading channels.

\section{A $G I|G| \infty$ model for broadcast percolation in the presence of channel randomness}

By applying much the same methods used in the previous section we may get results for the broadcast percolation problem in one-dimensional ad hoc networks [24], defined as follows.

\footnotetext{
${ }^{2}$ With the usual notation, $f(n)=o(g(n))$ if $\lim _{n \rightarrow+\infty} \frac{f(n)}{g(n)}=0$.
}

Consider a message generated by a node at $X_{0}=0$. The message is intended to be received by all the devices on the right of the source node (in this sense it is a broadcast message). We want to know how many nodes we can reach before the message stops propagating and how far (in terms of distance) our broadcast message can go.

It is easy to see that, in the presence of a deterministic channel model, broadcast percolation and connectivity are the same problem. In the case of broadcast percolation, we may extend the framework to take into account the randomness inherently present in the radio channel, which, in turn, translates into a statistical characterization of the transmission range $R$. In this case, in general, no results can be drawn from the analysis in terms of connectivity. Indeed, since the transmission range is not deterministic any longer, in principle nothing may be inferred in terms of the backward direction.

In any case, we may get results for general transmission range distribution and general node placement statistics, by applying results on the busy periods of the $G I|G| \infty$ queue [20]. Note that, since we assume that a single message will be present in the network at any time, no interference issues arise in this framework ${ }^{3}$.

\subsection{Characterizing the communication range statistics} in the presence of fading

The notion of "communication range", which had an immediate physical interpretation in the case of a deterministic channel model, becomes in this framework only a random variable whose distribution characterizes the capacity of any node to percolate a broadcast message. Let us start with the case of Rayleigh fading. We assume that the channel is characterized by flat slow fading, with no line-of-sight (this clearly represents a pessimistic assumption). In Rayleigh fading channels, the impact of the randomness due the Gaussian noise is usually negligible compared to the variation in SNR due to the fading process. We assume that the fading is constant over the transmission of a frame and subsequent fadings are iid (block-fading channel). Hence, taking the fading power equal to 1 , the average SNR may be written as $\bar{\gamma}=\frac{P_{t x}}{d^{\alpha} P_{n o i s e}}$. The pdf of the SNR is given by [26]:

$f_{\gamma}(a)=\frac{1}{\bar{\gamma}} e^{-\frac{a}{\bar{\gamma}}}=\frac{P_{\text {noise }} d^{\alpha}}{P_{t x}} e^{-\frac{a d^{\alpha} P_{n o i s e}}{P_{t x}}}$.

\footnotetext{
${ }^{3}$ From an implementation perspective, our assumptions may be used to model either a system where transmitters are using orthogonal channels (either in the time, frequency or code domain) or the traffic load in the network is very low or an efficient collision resolution protocol is adopted. It should thus be clear that our assumptions lead to optimistic results with respect to some real-world situations.
} 
In our model, the transmitted message can be correctly decoded if and only if the SNR $\gamma$ is greater than a given threshold $\Psi$. The probability that the message is correctly received at a distance $d$ is given by:

$P[\gamma(d) \geq \Psi]=\int_{\Psi}^{\infty} f_{\gamma}(a) \mathrm{d} a=e^{-\frac{\Psi}{\bar{\gamma}}}=e^{-\frac{d^{\alpha} \Psi P_{\text {noise }}}{P_{t x}}}$.

The transmission range statistics may then be obtained:

$$
\begin{aligned}
F_{R}(a) & =P[R \leq a]=1-P[R>a] \\
& =1-P[\gamma(a)>\Psi]=1-e^{-\frac{a^{\alpha} \Psi P_{\text {noise }}}{P_{t x}}} .
\end{aligned}
$$

The average transmission range may be computed as [27]:

$$
\begin{aligned}
E[R] & =\int_{0}^{+\infty}\left[1-F_{R}(a)\right] \mathrm{d} a=\int_{0}^{+\infty} e^{-\frac{a^{\alpha} \Psi P_{\text {noise }}}{P_{t x}}} \mathrm{~d} a \\
& =\frac{\Gamma\left(\alpha^{-1}\right)}{\alpha}\left(\frac{P_{t x}}{\Psi P_{\text {noise }}}\right)^{\frac{1}{\alpha}},
\end{aligned}
$$

where $\Gamma(\cdot)$ is the usual Gamma function.

Another important effect is the so-called shadowing [28], which is usually described by means of a lognormal distribution (i.e. the received signal power, in $\mathrm{dB}$, is normally distributed around its mean, which is given by the path loss). In particular, we will show that the performance enhancements in the presence of lognormal shadowing, observed independently by [29] and [14] for a two-dimensional network, hold also in the one-dimensional case. The received power is lognormally distributed; the communication range distribution is given by:

$$
\begin{aligned}
F_{R}(a) & =1-P[R>a]=1-P[\gamma(a)>\Psi] \\
& =1-\int_{\frac{\Psi P_{\text {noise }}}{P_{t x}}}^{+\infty} \mathrm{d} x \frac{1}{\sqrt{2 \pi} \sigma x} e^{-\frac{1}{2}\left(\frac{\ln x-\ln \left(a^{-\alpha}\right)}{\sigma}\right)^{2}} .
\end{aligned}
$$

Setting $t=\frac{\ln \frac{x}{a^{-\alpha}}}{\sigma}$, we get:

$$
\begin{aligned}
F_{R}(a) & =1-\int_{\frac{\ln }{P_{t x} a^{-\alpha}}}^{+\infty} \frac{\mathrm{d} t}{\sqrt{2 \pi}} e^{-\frac{t^{2}}{2}} \\
& =1-Q\left(\frac{\ln \frac{\Psi P_{\text {noise }} a^{\alpha}}{P_{t x}}}{\sigma}\right)=\Phi\left(\frac{\ln \frac{\Psi P_{\text {noise }} a^{\alpha}}{P_{t x}}}{\sigma}\right) .
\end{aligned}
$$

It is worth noting that a stochastic ordering among the distributions corresponding to various values of $\sigma$ is possible ${ }^{4}$,

\footnotetext{
${ }^{4}$ Given two random variables $A$ and $B$, we say that $A$ is stochastically smaller than $B, A \preceq B$ if $F_{A}(x) \geq F_{B}(x) \forall x \geq 0$ [30].
}

and we can write:

$R_{\sigma_{1}} \preceq R_{\sigma_{2}}, \quad \sigma_{1} \leq \sigma 2$,

so that we have a clear formalization of the beneficial impact of lognormal shadowing 5 .

The mean communication range is in this case given by:

$$
\begin{aligned}
E[R] & =\int_{0}^{+\infty} \mathrm{d} a \int_{\frac{\ln \Psi P_{\text {noise }}-\ln P_{t x}-\ln \left(a^{-\alpha}\right)}{\sigma}}^{+\infty} \frac{\mathrm{d} t}{\sqrt{2 \pi}} e^{-\frac{t^{2}}{2}} \\
& =\int_{-\infty}^{+\infty} \mathrm{d} t \int_{0}^{\left(\frac{e^{t \sigma} P_{t x}}{\Psi P_{n o i s e}}\right)^{\frac{1}{\alpha}}} \mathrm{d} a \frac{1}{\sqrt{2 \pi}} e^{-\frac{t^{2}}{2}} \\
& =\left(\frac{P_{t x}}{\Psi P_{\text {noise }}}\right)^{\frac{1}{\alpha}} \int_{-\infty}^{+\infty} \frac{\mathrm{d} t}{\sqrt{2 \pi}} e^{-\frac{t^{2}-\frac{2 t \sigma}{\alpha}}{2}} \\
& =\left(\frac{P_{t x}}{\Psi P_{\text {noise }}}\right)^{\frac{1}{\alpha}} e^{\frac{\sigma^{2}}{2 \alpha^{2}}} .
\end{aligned}
$$

Next, the case of superimposed lognormal shadowing and Rayleigh fading can be considered, leading to the following characterization of the communication range statistics:

$$
\begin{aligned}
F_{R}(a) & =1-P[R>a]=1-P[\gamma(a)>\Psi] \\
& =1-\int_{-\infty}^{+\infty} \mathrm{d} x e^{-\frac{\Psi P_{\text {noise }}}{P_{t x} x}} \frac{1}{\sqrt{2 \pi} \sigma x} e^{-\frac{1}{2}\left(\frac{\ln x-\ln \left(a^{-\alpha}\right)}{\sigma}\right)^{2}} \\
& =1-e^{-\frac{\Psi P_{\text {noise }}}{P_{t x}}} \int_{-\infty}^{+\infty} \mathrm{d} t e^{-e^{\sigma t} a^{-\alpha}} \frac{e^{-\frac{t^{2}}{2}}}{\sqrt{2 \pi}} .
\end{aligned}
$$

The mean communication range may be computed proceeding as above and using some results from [27]. After some algebra, we get:

$E[R]=\left(\frac{P_{t x}}{\Psi P_{\text {noise }}}\right)^{\frac{1}{\alpha}} e^{\frac{\sigma^{2}}{2 \alpha^{2}}} \frac{\Gamma\left(\alpha^{-1}\right)}{\alpha}$.

Note that, since $y \Gamma(y) \leq 1 \forall y \leq 1$, Rayleigh fading has a negative impact on the mean broadcast percolation distance.

\subsection{A special case: $M|G| \infty$}

In order to gain more insight into the impact on network connectivity of channel randomness, we focus on the simplest case, assuming that the nodes are distributed along the semi-infinite line according to a Poisson point process with intensity $\lambda$. While this is clearly just one of the possible distributions, it enables us to get closed-form expression for

\footnotetext{
${ }^{5}$ Note, however, that such results do not account for the very basic fact that a channel, in reality, cannot amplify a signal, so that its real impact on the network connectivity in a more realistic setting is not clear yet.
} 
the broadcast percolation distance statistics, thus allowing an in-depth analysis of the impact of shadowing and fading phenomena. The transmission range is assumed to be characterized by means of (23), or, accordingly, to any given cdf $F_{R}(a)$. Hence, from [20] the LST of the busy period of the equivalent $M|G| \infty$ queue is given by:

$\mathcal{B}(s)=1+\frac{s}{\lambda}-\frac{1}{\lambda \mathcal{P}_{0}(s)}$,

where $\mathcal{P}_{0}(s)$ is the Laplace transform of the function:

$P_{0}(t)=e^{-\lambda \int_{0}^{t} P[R>a] \mathrm{d} a}=e^{-\lambda \int_{0}^{t}\left[1-F_{R}(a)\right] \mathrm{d} a}$,

representing the probability that at time $t$ the system is empty. Differentiating (31), we may easily obtain the following expression for the average broadcast percolation distance:

$E[B]=\frac{1}{\lambda P_{0}}-\frac{1}{\lambda}$,

where $P_{0}=\lim _{t \rightarrow+\infty} P_{0}(t)=e^{-\lambda E[R]}$. The expression above may then be rewritten as:

$E[B]=\frac{e^{\lambda E[R]}-1}{\lambda}$.

Hence the average broadcast percolation distance depends only on the average transmission range and not on its distribution. Hence we can get a first comparison between the four channel models (deterministic, Rayleigh fading, lognormal shadowing, superimposed fading and shadowing) in terms of the mean broadcast percolation distance.

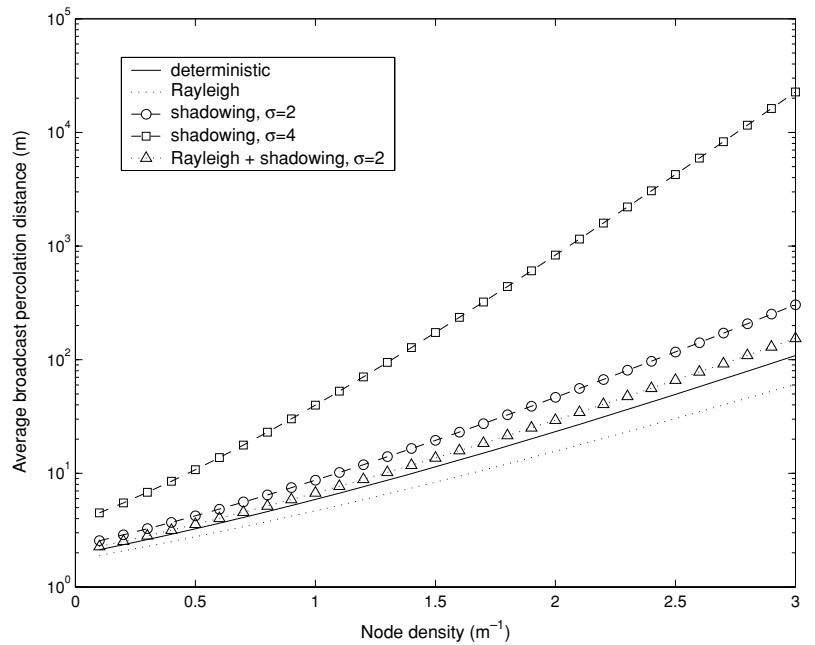

Fig. 7 Mean broadcast percolation distance for various channel models, $\alpha=3.5, \frac{P_{t x}}{\Psi P_{\text {noise }}}=10$

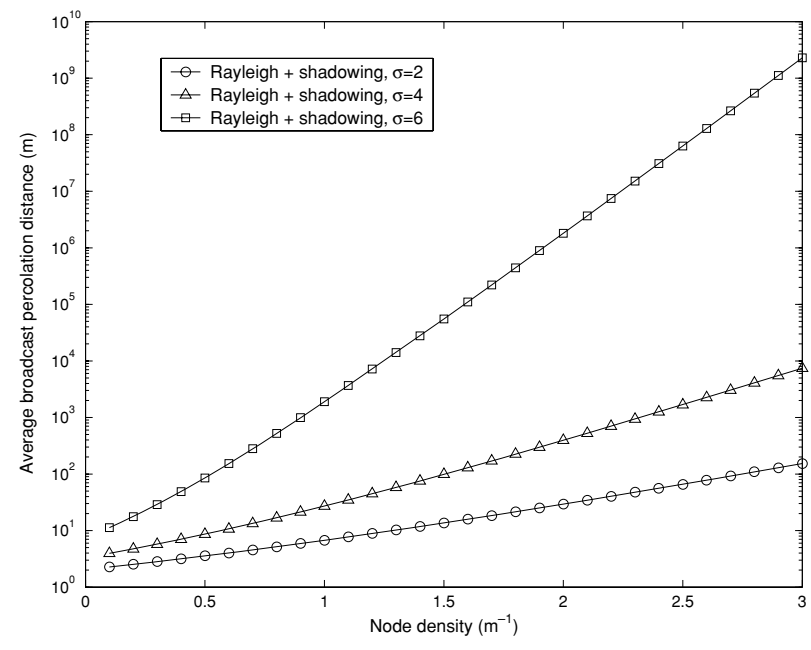

Fig. 8 Mean broadcast percolation distance for various values of $\sigma$, superimposed Rayleigh fading and lognormal shadowing, $\alpha=3.5$, $\frac{P_{t x}}{\Psi P_{\text {noise }}}=10$

As an example, we plotted in Fig. 7 the average broadcast percolation distance vs. the node density for various channel models. From the curves it is evident the positive effect of lognormal shadowing, as well as the (small) negative effect of Rayleigh fading. Some more results are reported in Fig. 8, where the superposition of lognormal shadowing and Rayleigh fading is considered. Notice that the larger the node density, the larger the difference between the curves corresponding to different values of the spread factor $\sigma$.

Another possible approach to the study of the broadcast percolation distance statistics is that of [22], obtaining the following expression for the busy period cdf:

$F_{B}(a)=1-\frac{\sum_{n=1}^{\infty} f^{* n}(a)}{\lambda}$,

where $f^{* n}(\cdot)$ denotes the $n$-fold convolution of $f(a)=$ $\lambda e^{-a^{\alpha} \beta} P_{0}(a)$. Hence, we may substitute in (35) and solve numerically, in order to get the statistics of the distance a broadcast message will travel. The analysis for the number of hosts reached by the broadcast is extremely difficult (see the note in the appendix of [22] for more details).

\subsection{Heavy traffic analysis for dense networks}

As done for the $M|D| \infty$ case, we can apply heavy traffic limits in order to study the behavior of broadcast percolation in dense networks. Let us assume that nodes are placed upon a semi-infinite line according to a Poisson point process of intensity $\lambda$, and that the channel model gives rise to a random transmission range with $\mathrm{cdf} F_{R}(\cdot)$. We recall the following result from [23]: 
Theorem 4. If $E\left[R^{2}\right]$ is finite, then as $\lambda \rightarrow+\infty$,

$$
F_{B}(a E[B]) \rightarrow 1-e^{-a}, a>0 .
$$

Note that the condition stated above is only sufficient; a necessary and sufficient condition is reported in [23]. Let us consider Rayleigh fading channels; then, by using results from [27]:

$$
\begin{aligned}
E\left[R^{2}\right] & =\int_{0}^{+\infty} \alpha a^{\alpha+1} e^{-\frac{a^{\alpha} \Psi P_{\text {noise }}}{P_{t x}}} \mathrm{~d} a \\
& =\frac{2}{\alpha} \Gamma\left(\frac{2}{\alpha}\right)\left(\frac{\Psi P_{\text {noise }}}{P_{t x}}\right)^{-\frac{2}{\alpha}}<+\infty .
\end{aligned}
$$

Similarly, some cumbersome algebra leads to the following analysis for the case of lognormal shadowing:

$$
\begin{aligned}
E\left[R^{2}\right] & =\int_{0}^{+\infty} 2 a\left[1-F_{R}(a)\right] \mathrm{d} a \\
& =\int_{0}^{+\infty} 2 a Q\left(\frac{\ln \frac{\Psi P_{\text {noise }} a^{\alpha}}{P_{t x}}}{\sigma}\right) \mathrm{d} a \\
& =\left(\frac{\Psi P_{\text {noise }}}{P_{t x}}\right)^{-\frac{2}{\alpha}} e^{\left(\frac{\sqrt{2} \sigma}{\alpha}\right)^{2}}<+\infty .
\end{aligned}
$$

For the case of superimposed fading and shadowing, we obtain:

$$
\begin{aligned}
E\left[R^{2}\right] & =\int_{0}^{+\infty} 2 a\left[1-F_{R}(a)\right] \mathrm{d} a \\
& =\frac{2}{\alpha} \Gamma\left(\frac{2}{\alpha}\right)\left(\frac{\Psi P_{\text {noise }}}{P_{t x}}\right)^{-\frac{2}{\alpha}} e^{\left(\frac{\sqrt{2} \sigma}{\alpha}\right)^{2}}<+\infty .
\end{aligned}
$$

For all the channel models considered, we may thus apply the proposition above, obtaining the following theorem:

Theorem 5. Consider a one-dimensional ad hoc network, with nodes placed according to a Poisson process of intensity $\lambda$, with a channel characterized by a path loss of the form $\left(\frac{1}{d}\right)^{\alpha}$ and by Rayleigh fading/lognormal shadowing/superimposed fading and shadowing. Assume that all nodes transmit at a fixed power $P_{t x}(\lambda)=\xi\left[\frac{\ln \lambda+c(\lambda)}{\lambda}\right]^{\alpha}$, where:

$\xi=\Psi P_{\text {noise }}\left(\frac{\alpha}{\Gamma\left(\alpha^{-1}\right)}\right)^{\alpha} \quad$ Rayleigh fading

$\xi=\Psi P_{\text {noise }} e^{-\frac{\sigma^{2}}{2 \alpha}} \quad$ lognormal shadowing;

$\xi=\Psi P_{\text {noise }} e^{-\frac{\sigma^{2}}{2 \alpha}}\left(\frac{\alpha}{\Gamma\left(\alpha^{-1}\right)}\right)^{\alpha} \quad$ superimposed fading and shadowing.
Then, a broadcast message generated by a node placed at the origin percolates until distance $d$ as $\lambda \rightarrow+\infty$ iff $\lim _{\lambda \rightarrow+\infty} c(\lambda)=+\infty$.

Proof: The proof follows along the lines of Theorem 2 . Indeed, it is sufficient to notice that the expression is basically the same, apart from the fact that here we have to consider the average communication range. Then a necessary and sufficient condition for asymptotic connectivity is that, if $E[R(\lambda)]=\frac{\ln \lambda+c(\lambda)}{\lambda}, \lim _{\lambda \rightarrow+\infty} c(\lambda)=+\infty$. Substituting the expression of $P_{t x}$ in (24), (28) and (30), respectively, we get that $E[R(\lambda)]=\frac{\ln \lambda+c(\lambda)}{\lambda}$. The result then follows from Theorem 4, (34) and (37).

It is remarkable to note that the scaling law for $P_{t x}$ is insensitive to the presence of fading/shadowing (up to a multiplicative factor). This provides a precious guideline for network designers, in that it shows that the fundamental scaling law depends only on the path loss factor $\alpha$ and not on the presence of shadowing/fading phenomena.

3.4. Improving broadcast percolation distance in the presence of Rayleigh fading by using multiple antennas

In this section we aim at studying the impact on the broadcast percolation distance of two schemes which take advantage of the diversity provided by small-scale (Rayleigh) fading by using multiple antennas. For the time being, the use of multiple antennas for ad hoc networks does not represent a viable choice, due to the increase in cost, complexity and power consumption caused by the adoption of such solution. Nonetheless, it is of theoretical interest to see if, by exploiting the diversity provided by Rayleigh fading (which has been shown in the previous subsections to have a negative effect on the broadcast percolation distance statistics), we can actually outperform the performance attainable in the absence of fading.

In order to keep the analysis simple, we focus on a channel model where Rayleigh fading only is present; the analysis can nonetheless be easily extended to account for the presence of the underlying lognormal shadowing, leading to similar results. For the same reason, we limit our analysis to the case of nodes distributed according to a Poisson process, and take as a performance metric the mean broadcast percolation distance.

We assume that each node employs $H$ antennas at the receiver, and consider two signal processing schemes, namely best path selection (BPS) and maximal ratio combining (MRC). Note that the same performance may be attained by schemes which employ multiple antennas at the transmitter, or at both the transmitter/receiver (e.g. the well-known Alamouti's scheme [31]). However, the use of multiple antennas 
for the transmission of a signal requires an increase in the transmission power (in reality also the signal processing required by the use of diversity schemes at the receiver increases the power consumption of the devices).

In the case of BPS the signal with the best SNR among the $\mathrm{H}$ received is decoded. It is not difficult to see that the system can be studied by means of an equivalent $M^{X}|G| \infty$ model, where the equivalent burst size is equal to $H$. In this case, the busy period LST is given by [20]:

$\mathcal{B}(s)=1+\frac{s}{\lambda}-\frac{1}{\lambda \mathcal{P}_{0}(s)}$,

where $\mathcal{P}_{0}(s)$ is the LST of:

$P_{0}(t)=\exp \left\{-\lambda \int_{0}^{t} \mathrm{~d} x\left[1-\left(F_{R}(x)\right)^{H}\right]\right\}$.

Taking $t \rightarrow+\infty$, we get:

$$
\begin{aligned}
P_{0} & =\exp \left\{-\lambda \int_{0}^{+\infty} \mathrm{d} x\left[1-\left(1-e^{-\frac{a^{\alpha} \Psi P_{\text {noise }}}{P_{t x}}}\right)^{H}\right]\right\} \\
& =\exp \left\{\lambda \sum_{j=1}^{H}\left(\begin{array}{c}
H \\
j
\end{array}\right)(-1)^{j} \int_{0}^{+\infty} \mathrm{d} x e^{-\frac{a^{\alpha} \Psi P_{\text {noise }}}{P_{t x}}}\right\} \\
& =\exp \left\{\lambda \sum_{j=1}^{H}\left(\begin{array}{c}
H \\
j
\end{array}\right)(-1)^{j} \frac{\left(\frac{\Psi P_{\text {noise }} j}{P_{t x}}\right)^{-\frac{1}{\alpha}}}{\alpha} \Gamma\left(\alpha^{-1}\right)\right\},
\end{aligned}
$$

from which by means of (33) we get the mean broadcast percolation distance.

In the case of maximal ratio combining (MRC), the signals from the various antennas are combined in such a way to maximize the resulting SNR. In this case, the resulting channel becomes an $H$-Nakagami fading channel [26]. In $H$-Nakagami fading, the instantaneous signal-to-noise ratio pdf is given by [26]:

$f_{\gamma}(a)=\left(\frac{H}{\bar{\gamma}}\right)^{H} \frac{a^{(H-1)}}{\Gamma(H)} e^{-H \frac{a}{\bar{\gamma}}}$

where $\Gamma(\cdot)$ is the usual Gamma function and $\bar{\gamma}=\frac{P_{t x}}{d^{\alpha} P_{\text {noise }}}$ is the average SNR at distance $d$. Note that for $H=1$ we obtain the usual expression for Rayleigh fading. The probability that the message is correctly received at distance $d$ is given by:

$P[\gamma(d)>\Psi]=\int_{\Psi}^{+\infty} f_{\gamma}(a) \mathrm{d} a=\frac{\Gamma\left(H, \frac{H d^{\alpha} \Psi P_{\text {noise }}}{P_{t x}}\right)}{\Gamma(H)}$,

where $\Gamma(\cdot, \cdot)$ is the incomplete Gamma function [27]. Since in our case $H$ is an integer, the expression above simplifies to:

$$
\begin{aligned}
P & {[\gamma(d)>\Psi] } \\
& =e^{-\frac{H d^{\alpha} \Psi P_{\text {noise }}}{P_{t x}}} \sum_{i=0}^{H-1}\left(\frac{H d^{\alpha} \Psi P_{\text {noise }}}{P_{t x}}\right)^{i} \cdot \frac{1}{(i+1) !} .
\end{aligned}
$$

Thus, the cdf of the transmission range is given by:

$$
\begin{aligned}
F_{R}(a) & =1-P[\gamma(a)>\Psi] \\
& =1-e^{-\frac{H a^{\alpha} \Psi P_{\text {noise }}}{P_{t x}}} \sum_{i=0}^{H-1}\left(\frac{H a^{\alpha} \Psi P_{\text {noise }}}{P_{t x}}\right)^{i} \cdot \frac{1}{(i+1) !} .
\end{aligned}
$$

The computation of the average transmission range requires some algebra, leading to:

$$
\begin{aligned}
E[R] & =\int_{0}^{+\infty}\left[1-F_{R}(a)\right] \mathrm{d} a \\
& =\sum_{i=0}^{H-1}\left(\frac{H \Psi P_{\text {noise }}}{P_{t x}}\right)^{-\frac{1}{\alpha}} \frac{1}{\alpha(i+1) !} \Gamma\left(i+\frac{1}{\alpha}\right) .
\end{aligned}
$$

It is worth noticing that, for both BPS and MRC, stochastic orderings with respect to the number of receiving antennas may be obtained:

$R_{H_{1}} \preceq R_{H_{2}} \quad H_{1} \leq H_{2}$

In Fig. 9 we reported some results for the BPS algorithm, with $\alpha=3.5$ and $\frac{P_{t x}}{\Psi P_{\text {noise }}}=10$. In such conditions, it may be seen that with the use of two (or more) antennas, we may get better results than those achievable in absence of Rayleigh fading. Similar results are obtained for MRC, as reported

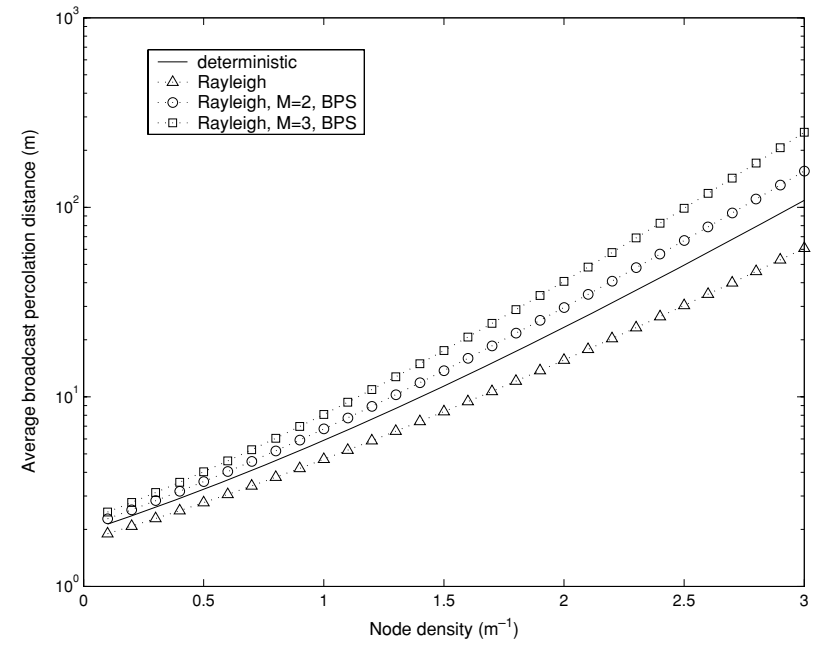

Fig. 9 Mean broadcast percolation distance for various values of $H$, Rayleigh fading, best path selection, $\alpha=3.5, \frac{P_{t x}}{\Psi P_{\text {noise }}}=10$ 


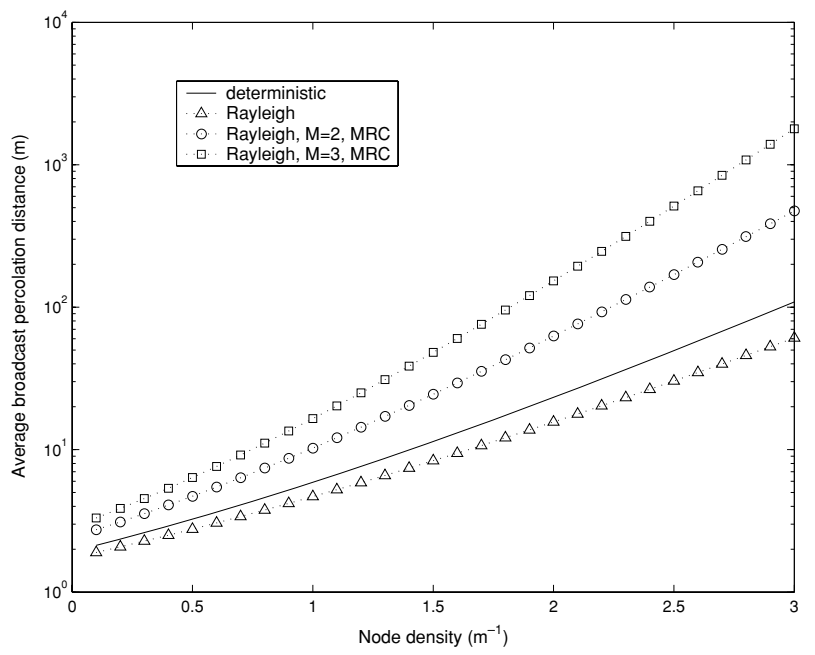

Fig. 10 Mean broadcast percolation distance for various values of $H$, Rayleigh fading, maximal ratio combining, $\alpha=3.5, \frac{P_{t x}}{\Psi P_{\text {noise }}}=10$

in Fig. 10 for the same parameter values. Clearly, the more complex MRC scheme outperforms BPS, so that MRC with $H=2$ antennas shows better performance than BPS with $H=3$ antennas.

\section{Unreliable ad hoc networks}

In largely deployed sensor networks, it is mandatory to take into account for the possible unreliability of the devices. We consider, as above, a one-dimensional network, where the distance between successive devices is given by independent random variables with a common distribution $F_{Y}(\cdot)$, and each node is active with probability $p(\lambda)$, where $\lambda^{-1}=E[Y]$. We aim thus at generalizing the results obtained in the previous sections for the case of reliable nodes. In particular, we will focus on the asymptotic behavior, in order to find scaling laws for $p(\lambda)$ and $R(\lambda)$.

As a starting point, consider the interdistance between two successive active nodes, which will be denoted by $\tilde{Y}$. In particular, under our assumptions, $\tilde{Y}=\sum_{i=1}^{K} Y_{i}$, where $K$ is a geometrically distributed random variable, having $\operatorname{pmf} p_{K}(a)=P[K=a]=p(\lambda) \cdot(1-p(\lambda))^{a-1}, a=$ $1,2, \ldots$.

A special case arises when $Y$ is exponentially distributed with mean $\lambda^{-1}$. In that situation, we end up with a statistical sampling of a Poisson process, which is still a Poisson process. Hence, $F_{\tilde{Y}}(a)=1-e^{-\lambda p(\lambda) a}$. For this case, the generalization of the connectivity and broadcast percolation results is trivial. In the more general case, we have the following expression for the Laplace-Stjelties transform of $f_{\tilde{Y}}(\cdot)$ :

$\tilde{\mathcal{Y}}(s)=\frac{\mathcal{Y}(s) p(\lambda)}{1-(1-p(\lambda)) \mathcal{Y}(s)}$
Then, numerical inversion may be performed to get the pdf of $\tilde{Y}$ and the rest of the analysis follows accordingly.

It is thus evident that the analysis of the connectivity of unreliable networks represents a straightforward generalization of what done in the previous sections for reliable nodes. However, some interesting phenomena do arise in the asymptote. Let us consider nodes distributed according to a Poisson point process of intensity $\lambda$, which are active with a given probability $p(\lambda)$. The reason for keeping $p$ as a function of $\lambda$ comes from some possible engineering tradeoff between the reliability of the devices and their number, in order to take advantage of economies of scale. In any case, the resulting process is still Poisson with intensity $\lambda p(\lambda)$. The problem comes from the fact that, from a network designer point of view, $p(\lambda)$ cannot be assumed to be known a priori. As a consequence, the transmission power (and consequently the transmission range) has to be dimensioned relying only on the knowledge of $\lambda$, a parameter that can be controlled in the deployment phase. In this case, even for a fixed $p(\lambda)$, a different scaling law for $R(\lambda)$ is necessary to provide asymptotic connectivity. The results of asymptotic analysis may be successfully employed as guidelines for the dimensioning of robust dense ad hoc networks.

As previously stated, we limit ourselves to the case of nodes distributed according to a Poisson point process of intensity $\lambda$, having deterministic communication range $R$, and study scaling laws as the density $\lambda \rightarrow+\infty$. For $\lim _{\lambda \rightarrow+\infty} p(\lambda)=p>0$, the condition found for the connectivity in reliable networks is clearly only necessary. A stronger condition for sufficiency is provided. The case $p(\lambda) \rightarrow 0$ is also investigated, with particular attention for the case $p(\lambda)=\lambda^{-\gamma}$. The results we will find closely resemble those obtained by Shakkottai et al. [19] for regular sensor grids.

Theorem 6. For a general $R(\lambda)$ with nonzero support, a necessary and sufficient condition for asymptotic connectivity at any finite distance $d$ is:

$\lim _{\lambda \rightarrow+\infty} \lambda p(\lambda) e^{-\lambda p(\lambda) R(\lambda)}=0$.

Proof: The probability of being connected at distance $d$ is given by:

$p_{C}(d)=e^{-\frac{d \lambda p(\lambda)}{e^{\lambda p(\lambda) R(\lambda)}-1}}$.

Then, we may study the behavior of the function $f(\lambda)$ :

$f(\lambda)=\frac{\lambda p(\lambda)}{e^{\lambda p(\lambda) R(\lambda)}-1}$. 
A necessary and sufficient condition for asymptotic connectivity is that $\lim _{\lambda \rightarrow+\infty} f(\lambda)=0$. The statement follows straightforwardly.

Now we consider a particular expression for $R(\lambda)$ and a finite non-zero $p$.

Theorem 7. If $p=\lim _{\lambda \rightarrow+\infty} p(\lambda)$ satisfies $0<p<1$ and $R(\lambda)=\frac{\ln \lambda+c(\lambda)}{\lambda}$, the network is asymptotically connected at distance $d$ if $c(\lambda)$ satisfies

$c(\lambda) \geq \zeta \ln \lambda$,

where $\zeta>\frac{1-p}{p}$. Furthermore, the network is asymptotically connected only if $c=\lim _{\lambda \rightarrow+\infty} c(\lambda)=+\infty$.

Proof: We start with the proof of the sufficient condition. From Theorem 6, considering the particular expression of $R(\lambda)$, we obtain:

$$
\begin{array}{r}
\lim _{\lambda \rightarrow+\infty} p(\lambda) \lambda^{1-p(\lambda)} e^{-p(\lambda) c(\lambda)}=\lim _{\lambda \rightarrow+\infty} p \lambda^{1-p} e^{-p c(\lambda)} \\
\leq \lim _{\lambda \rightarrow+\infty} p \lambda^{1-p} e^{-p \zeta \ln \lambda}=\lim _{\lambda \rightarrow+\infty} p \lambda^{1-p-p \zeta},
\end{array}
$$

which clearly converges to 0 for $\zeta>\frac{1-p}{p}$.

The necessary condition is proven by reductio ad absurdum. Let us assume $c<+\infty$. Then,

$\lim _{\lambda \rightarrow+\infty} f(\lambda)=\lim _{\lambda \rightarrow+\infty} p \lambda^{1-p} e^{-p c}$,

which clearly diverges, and the network is not asymptotically connected.

Note that there is a big gap between the necessary and sufficient condition.

As done in [19] let us consider a particular case of $p(\lambda)$ and a general $R(\lambda)$.

Theorem 8. Let $p(\lambda)$ be $p(\lambda)=\lambda^{-\gamma}$, where $0<\gamma<1$. Then a sufficient condition for asymptotic network connectivity at distance $d$ is given by:

$p(\lambda) R(\lambda) \geq \zeta \frac{\ln \lambda}{\lambda}$,

where the constant $\zeta$ satisfies $\zeta>1-\gamma$.

Proof: From (51), applying (55) we have:

$f(\lambda) \leq \frac{\lambda p(\lambda)}{e^{\zeta \ln \lambda}-1}=\frac{\lambda p(\lambda)}{\lambda^{\zeta}-1} \sim \lambda^{1-\zeta} p(\lambda)=\lambda^{1-\zeta-\gamma}$,

which tends to 0 iff $\gamma>1-\zeta$.
Considering a particular expression for $R(\lambda)$, the previous result may be specified as follows:

Corollary 1. Let $p(\lambda)$ be $p(\lambda)=\lambda^{-\gamma}$, where $0<\gamma<1$ and $R(\lambda)=\frac{\ln \lambda+c(\lambda)}{\lambda}$. Then, a sufficient condition for asymptotic connectivity is that

$c(\lambda) \geq\left(\zeta \lambda^{\gamma}-1\right) \ln \lambda$

where $\zeta$ satisfies $\zeta>1-\gamma$.

Proof: Under the condition (57), we clearly have:

$p(\lambda) R(\lambda)=\frac{\ln \lambda+c(\lambda)}{\lambda^{1+\gamma}} \geq \frac{\zeta \lambda^{\gamma} \ln \gamma}{\lambda^{1+\gamma}}=\frac{\zeta \ln \gamma}{\lambda}$,

and Theorem 8 may be applied to conclude the proof.

A necessary condition may also be given:

Proposition 2. Let $p(\lambda)$ be $p(\lambda)=\lambda^{-\gamma}$, where $0<\gamma<1$ and $R(\lambda)=\frac{\ln \lambda+c(\lambda)}{\lambda}$. Then, a necessary condition for asymptotic connectivity is that $c=\lim _{\lambda \rightarrow+\infty} c(\lambda)=+\infty$.

Proof: The proof follows by a reductio ad absurdum argument. Let us assume $c<+\infty$ and consider the limiting behavior of $f(\lambda)$ :

$$
\begin{aligned}
\lim _{\lambda \rightarrow+\infty} f(\lambda) & =\lim _{\lambda \rightarrow+\infty} \lambda^{1-\gamma} e^{-\lambda^{-\gamma}[\ln \lambda+c(\lambda)]} \\
& =\lim _{\lambda \rightarrow+\infty} \lambda^{1-\gamma} e^{-\frac{\ln \lambda}{\lambda^{\gamma}}} e^{-\frac{c(\lambda)}{\lambda^{\gamma}}}
\end{aligned}
$$

Now, if $c<+\infty$, we have

$f(\lambda) \sim \lambda^{1-\gamma}$,

which diverges, and hence the network is not asymptotically connected.

While the results above were derived for a deterministic channel model, similar scaling laws hold also for broadcast percolation in fading channels. In particular, in the presence of Rayleigh fading, lognormal shadowing or a combination of the two and assuming that nodes are distributed according to a Poisson point process, the following results hold.

Theorem 9. For a general $P_{t x}(\lambda)$, a necessary and sufficient condition for a broadcast message to asymptotically percolate to distance $d$ is that:

$\lim _{\lambda \rightarrow+\infty} \lambda p(\lambda) e^{-\lambda p(\lambda)\left[P_{t x}(\lambda)\right]^{\frac{1}{\alpha}}}=0$. 
Theorem 10. If $p=\lim _{\lambda \rightarrow+\infty} p(\lambda)$ satisfies $0<p<1$ and $P_{t x}(\lambda)=\left(\frac{\ln \lambda+c(\lambda)}{\lambda}\right)^{\alpha}$, a broadcast message asymptotically percolates to distance $d$ if $c(\lambda)$ satisfies

$c(\lambda) \geq \zeta \ln \lambda$,

where $\zeta>\frac{1-p}{p}$. Furthermore, the network asymptotically supports broadcast percolation only if $c=\lim _{\lambda \rightarrow+\infty} c(\lambda)=$ $+\infty$.

Theorem 11. Let $p(\lambda)$ be $p(\lambda)=\lambda^{-\gamma}$, where $0<\gamma<1$. Then a sufficient condition for a broadcast message to asymptotically percolate to distance $d$ is given by:

$p(\lambda)\left(P_{t x}(\lambda)\right)^{\frac{1}{\alpha}} \geq \zeta \frac{\ln \lambda}{\lambda}$,

where the constant $\zeta$ satisfies $\zeta>1-\gamma$.

Corollary 2. Let $p(\lambda)$ be $p(\lambda)=\lambda^{-\gamma}$, where $0<\gamma<1$ and $P_{t x}(\lambda)=\left(\frac{\ln \lambda+c(\lambda)}{\lambda}\right)^{\alpha}$. Then, a sufficient condition for a broadcast message to asymptotically percolate is that

$c(\lambda) \geq\left(\zeta \lambda^{\gamma}-1\right) \ln \lambda$

where $\zeta$ satisfies $\zeta>1-\gamma$.

Proposition 3. Let $p(\lambda)$ be $p(\lambda)=\lambda^{-\gamma}$, where $0<\gamma<1$ and $P_{t x}(\lambda)=\left(\frac{\ln \lambda+c(\lambda)}{\lambda}\right)^{\alpha}$. Then, a necessary condition for a broadcast message to asymptotically percolate is that $c=$ $\lim _{\lambda \rightarrow+\infty} c(\lambda)=+\infty$

\section{Conclusions}

In this paper we have presented some novel results, obtained by means of queueing theoretical tools, on connectivity issues in one-dimensional wireless ad hoc networks. For a deterministic channel model, we have analyzed the influence of the nodes placement statistics on some metrics of interest, such as the coverage probability, the node isolation probability, the mean cluster size and the probability of the generic $k$-th node to be connected, showing how heavy-tailed distributions may degrade the performance in highly dense networks.

The similar problem of broadcast percolation has been addressed by means of an equivalent $G I|G| \infty$ queueing system. For this case, we have extended the framework to account for a random communication range, such as the one induced by Rayleigh fading and lognormal shadowing. In the presence of channel randomness, in general, no connectivity results may be obtained. Nevertheless results can be inferred under two different assumptions: (i) the propagation in the backward and forward directions is subject to independent fading/shadowing phenomena;

(ii) the fading/shadowing in the backward and forward directions is the same.

Indeed, under assumption (i) it is easy to see that connectivity results may be drawn by studying the broadcast percolation distance in a network where the probability that the range is greater than $a$ is the square of the probability that the one-direction range is greater than $a$. Under assumption (ii), on the other hand, connectivity at distance $d$ and capability of percolating a message until distance $d$ coincide.

We have also addressed the possibility of exploiting the diversity provided by Rayleigh fading in order to enhance network connectivity. Results have been obtained, in terms of stochastic orderings with respect to the number of antennas, for two popular signal processing schemes, BPS and MRC. It has been shown that, by using multiple antennas, it is possible to overcome the performance achievable in the absence of fading.

For the case of nodes distributed according to a Poisson point process, we have showed how heavy traffic theorems for the equivalent queueing model may be applied to derive scaling laws for the transmission power in the ad hoc network, in order to achieve asymptotic connectivity and broadcast percolation capability. Finally, the framework has been extended to account for unreliable nodes, with a particular emphasis on the asymptotic behavior of such networks, which shows a noteworthy difference with respect to the fully reliable case.

Two research directions appear of major interest, namely the evaluation of the impact of cooperation mechanisms (in which multiple nodes simultaneously transmit the same message in a coherent fashion) and the extension to a more general stationary ergodic framework, along the lines of [32].

\section{References}

1. P. Gupta and P. R. Kumar, "Critical power for asymptotic connectivity," in Proc. of IEEE CDC, Tampa, USA, 1998.

2. P. Santi and D. M. Blough, "The critical transmitting range for connectivity in sparse wireless ad hoc networks," IEEE Trans. on Mob. Comp., vol. 2, no. 1, pp. 25-39, Jan-Mar 2003.

3. O. Dousse, P. Thiran, and M. Hasler, "Connectivity in ad-hoc and hybrid networks," in Proc. of IEEE INFOCOM, New York, USA, 2002.

4. O. Dousse, F. Baccelli, and P. Thiran, "Impact of interferences on connectivity in ad-hoc networks," in Proc. of IEEE INFOCOM, San Francisco, USA, 2003.

5. P. Gupta and P. R. Kumar, "The capacity of wireless networks," IEEE Trans. on Inf. Th., vol. 46, no. 2, pp. 388-404, Mar. 2000.

6. A. E. Gamal, J. Mammen, B. Prabhakar, and D. Shah, "Throughputdelay trade-off in wireless networks," in Proc. of IEEE INFOCOM, Hong Kong, 2004. 
7. S. R. Kulkarni and P. Viswanath, "A deterministic approach to throughput scaling in wireless networks." IEEE Trans. on Inf. Th., vol. 50, no. 6, pp. 1041-1049, Jun. 2004.

8. A. Rajeswaran and R. Negi, "Capacity of power constrained ad-hoc networks," in Proc. of IEEE INFOCOM, Hong Kong, 2004.

9. A. Jovicic, P. Viswanath, and S. R. Kulkarni, "Upper bounds to transport capacity of wireless networks," IEEE Trans. on Inf. Th. vol. 50, no. 11, pp. 2555-2565, Nov. 2004.

10. M. Franceschetti, O. Dousse, D. Tse, and P. Thiran, "On the throughput capacity of random wireless networks," 2004, subm. for publ.

11. O. Dousse and P. Thiran, "Connectivity vs capacity in dense ad hoc networks," in Proc. of IEEE INFOCOM, Hong Kong, 2004.

12. M. Desai and D. Manjunath, "On the connectivity in finite ad hoc networks," IEEE Comm. Lett., vol. 6, no. 10, pp. 437-439, Oct 2002.

13. P. Panchapakesan and D. Manjunath, "On the transmission range in dense ad hoc radio networks," in Proc. of SPCOM, Bangalore, India, 2001.

14. R. Hekmat and P. Van Mieghem, "Study of connectivity in wireless ad-hoc networks with an improved radio model," in Proc. of WiOpt, Cambridge, UK, 2004.

15. M. Franceschetti, L. Booth, J. Bruck, M.Cook, and R. Meester, "Continuum percolation with unreliable and spread out connections," Journal of Statistical Physics, vol. 118, no. 3/4, pp. 721-734, Feb. 2005.

16. D. Miorandi and E. Altman, "Coverage and connectivity of adhoc networks in presence of channel randomness," INRIA, Tech. Rep. RR5377, 2004. [Online]. Available: http://www.inria.fr/rrrt/ rr-5377.html

17. P. Santi, "The critical transmitting range for connectivity in mobile ad hoc networks," IEEE Trans. on Mob. Comp., vol. 4, no. 2, pp. 310-317, Mar. 2005.

18. P. Hall, Introduction to the theory of coverage processes. New York: J. Wiley and sons, 1988.

19. S. Shakkottai, R. Srikant, and N. B. Shroff, "Unreliable sensor grids: coverage, connectivity and diameter," in Proc. of IEEE INFOCOM, San Francisco, CA, 2003.

20. L. Liu and D.-H. Shi, "Busy period in $G I^{X}|G| \infty$," J. Appl. Prob., vol. 33, pp. 815-829, 1996.

21. J. Abate, G. L. Choudhury, and W. Whitt, "An introduction to numerical transform inversion and its application to probability models," in Computational Probability, W. Grassman, Ed. Boston: Kluwer, 1999, pp. 257-323.

22. W. Stadje, "The busy period of the queueing system $M|G| \infty$," J. Appl. Prob., vol. 22, pp. 697-704, 1985.

23. P. Hall, "Heavy traffic approximations for busy period in an $M|G| \infty$ queue," Stochastic Processes and their Applications, vol. 19, pp. 259-269, 1985.

24. Y.-C. Cheng and T. G. Robertazzi, "Critical connectivity phenomena in multihop radio models," IEEE Trans.Comm., vol. 37, no. 7, pp. 770-777, Jul 1989.

25. F. Baccelli and P. Bremaud, Elements of Queueing Theory. Berlin: Springer-Verlag, 1994.

26. L. Hanzo, C. H. Wong, and M. S. Yee, Adaptive Wireless Transceivers. New York: John Wiley and Sons, 2002.

27. I. S. Gradshteyn and I. M. Ryzhik, Table of integrals, series, and products. Orlando: Academic Press, 1983.

28. T. S. Rappaport, Wireless Communications. Upper Saddle River, New Jersey: Prentice Hall, 1999.

29. C. Bettstetter and C. Hartmann, "Connectivity of wireless multihop networks in a shadow fading environment," in Proc. of ACM MSWiM, San Diego, CA, 2003.

30. D. Stoyan, Comparison Methods for Queues and Other Stochasti Models. New York: John Wiley \& Sons, 1983.

31. S. M. Alamouti, "A simple transmit diversity technique for wireless communications," IEEE J. Sel. Areas on Comm., vol. 16, no. 8, pp. 1451-1458, Oct. 1998.

32. E. Altman, "On stochastic recursive equations and infinite server queues," in Proc. of IEEE INFOCOM, Miami, 2005.

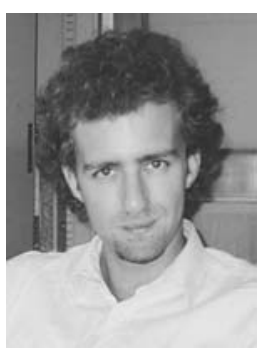

wireless networks.

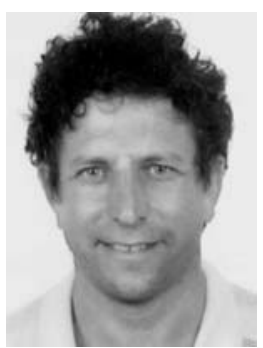

Daniele Miorandi received his "Laurea" (summa cum laude) and Ph.D. degrees from Univ. of Padova (Italy) in 2001 and 2005, respectively. He currently holds a post-doc position at CREATE-NET, Trento (Italy). In 2003/04 he spent 12 months of his doctoral thesis visiting the MAESTRO team at INRIA Sophia Antipolis (France). His research interests include stochastic modelling, performance evaluation and protocols design for

Eitan Altman received the B.Sc. degree in electrical engineering (1984), the B.A. degree in physics (1984) and the Ph.D. degree in electrical engineering (1990), all from the Technion-Israel Institute, Haifa. In (1990) he further received his B.Mus. degree in music composition in Tel-Aviv university. Since 1990, he has been with INRIA (National research institute in informatics and control) in Sophia-Antipolis, France. His current research interests include performance evaluation and control of telecommunication networks and in particular congestion control, wireless communications and networking games. He is in the editorial board of several scientific journals: Stochastic Models, JEDC, COMNET, SIAM SICON and WINET. He has been the (co)chairman of the program committee of several international conferences and workshops (on game theory, networking games and mobile networks). More informaion can be found at http://www.inria.fr/mistral/personnel/Eitan.Altman/me.html 